\title{
Salt and Pepper Noise Detection and Removal in Gray Scale Images: An Experimental Analysis
}

\author{
E.Jebamalar Leavline, D.Asir Antony Gnana Singh \\ Bharathidasan Institute of Technology, Anna University Chennai, Regional Centre \\ Tiruchirappalli- 620024 \\ jebi.lee@gmail.com,asirantony@gnail.com
}

\begin{abstract}
Impulse noise removal is a mechanism for detection and removal of impulse noise from images. Median filters are preferred for removing impulse noise because of their simplicity and less computational complexity. In this paper, impulse noise removal using the standard median filter and its variants are analyzed. Extensive simulations have been carried out on a set of standard gray scale images and the state of the art median filter variants are compared in terms of the well known image quality assessment metrics namely mean square error, peak signal to noise ratio and multiscale structural similarity index.
\end{abstract}

Keywords: Image denoising, impulse noise, impulse detector, spatial domain techniques, median filter

\section{Introduction}

Image noise removal plays a vital role in image processing as a pre-processing stage. The non-ideal imaging systems introduce potential degradations in digital images. Noise disturbances may also be caused by electronic imaging sensors, film granularity, and channel noise. High levels of noise are always undesirable; hence noise removal has to be employed before the image could be used for further analysis [1-3].

Salt and pepper noise is an impulse type of noise, which is also referred to as intensity spikes. This is caused generally due to dead pixels, analog-to-digital converter errors, errors in data transmission, malfunctioning of pixel elements in the camera sensors, faulty memory locations, or timing errors in the digitization process. It has only two possible values, 'a' and ' $b$ '. The probability of each is typically less than 1 . The corrupted pixels are set alternatively to the minimum or to the maximum intensity values, giving the image a "salt and pepper" like appearance. Unaffected pixels remain unchanged. For an 8-bit image, the typical intensity value for pepper noise is 0 and for salt noise 255 .

There are several impulse noise removal techniques found in literature. The median filter is one of the simplest and most popular approaches for impulse noise removal. However, the simple median filter fails to restore the images at considerably higher noise densities $[4,5]$. Adaptive median filter [6] works well at low noise densities. But at higher noise densities, it leads to larger window size which may blur the image. In switching median filter [7-9] the threshold value is predefined based on which the decision is made. But, obtaining optimal decision is difficult, also it ignores the local features of the image and hence edge details are not preserved at higher noise densities. The weighted median filter (WMF) is another variant of standard median filter with higher weight given to the pixel under inspection [10]. The adaptive center-weighted median (ACWM) [11, 12] filters have variable center weights to define a more general operator, which realizes the impulse detection using the differences 
defined between the outputs of CWM filters and the current pixel. The ultimate output is switched between the median and the current pixel itself. The tristate median filter (TMF) eliminates impulse noise by making decision on three states. It is a combination of standard median filter and center weighted median filter [13].

In this paper, an experimental study on the state of the art impulse noise removal techniques mentioned above is presented. The rest of the paper is organized as follows. Section 2 describes the process of impulse noise removal using standard median filter. Some of the variants of median filter are discussed in Section 3. The experimental results and analysis are presented in Section 4 and the paper is concluded in Section 5.

\section{Impulse Noise Removal using Median Filter}

The simple median filter replaces the center pixel of the window (Eg. 3X3.5X5 etc.) considered, by median of the window. If the center pixel is either ' 0 ' (Pepper) or '255' (Salt), it is replaced by median of the window which will be other than 0 or 255 . The major drawback of standard median filter is that even if the pixel under consideration is uncorrupted (other than 0 or 255), it is replaced by the median of the window. This will deteriorate the overall visual quality of the image. In addition, the simple median filter fails to preserve the edges. It works as follows. Window $=\{23,32,41,255,45,52,23,32,41\}$. The window sorted in ascending order $=\{23,23,32,32,41,41,45,52,255\}$. Median is the mid value after sorting i.e., 41. Hence the uncorrupted pixel is replaced by median of the window.

$$
\begin{aligned}
& \text { Sample window } \\
& {\left[\begin{array}{ccc}
23 & 32 & 41 \\
255 & (45) & 52 \\
23 & 32 & 41
\end{array}\right] \longrightarrow\left[\begin{array}{ccc}
23 & 32 & 41 \\
255 & (41) & 52 \\
23 & 32 & 41
\end{array}\right]}
\end{aligned}
$$

If the pixel under consideration is corrupted as shown below, the impulse noise will be removed following the same method [4].

$$
\begin{gathered}
\text { Sample window } \\
{\left[\begin{array}{ccc}
23 & 32 & 41 \\
255 & (255) & 52 \\
23 & 32 & 41
\end{array}\right] \longrightarrow\left[\begin{array}{ccc}
23 & 32 & 41 \\
255 & (41) & 52 \\
23 & 32 & 41
\end{array}\right]}
\end{gathered}
$$

\section{Median Filter Variants for Impulse Noise Removal}

\subsection{Adaptive Median Filter:}

The Adaptive Median Filter performs spatial processing to determine which pixels in an image have been affected by impulse noise. The Adaptive Median Filter classifies pixels as noise by comparing each pixel in the image to its surrounding neighbor pixels. The size of the neighborhood is adjustable, as well as the threshold for the comparison. A pixel that is different from a majority of its neighbors, as well as being not structurally aligned with those pixels to which it is similar, is labeled as impulse noise. These noisy pixels are then replaced by the median value of the pixels in the neighborhood that have passed the noise labeling test. Adaptive median filter changes the size of the neighborhood (window) during operation. The standard median filter does not perform well when the noise density is high, while the 
adaptive median filter can better handle these noises. Also, the adaptive median filter preserves image details such as edges and smooth non-impulsive noise, while the standard median filter does not [4-6].

\subsection{Switching Median Filter:}

It uses four 1-D Laplacian operators (Fig.1) that are sensitive to edges in a different orientation. Then, the minimum absolute value of these four convolutions $\left(r_{i j}\right)$ is used for impulse detection, which is represented as

$$
r_{i j}=\min \left\{\left|X_{i j} \otimes K_{p}\right|: p=1,2,3,4\right\}
$$

$\mathrm{r}_{\mathrm{ij}}$ is large when is corrupted by noise, and is small when is noise-free whether or not it is a flat-region, edge, or thin-line pixel. So, it can be compared with the threshold $\mathrm{T}$ to find whether the pixel is corrupted or not. This method prevents the removal of fine details such as thin lines from the images and thus provides improved impulse detection ability [7-9].

\begin{tabular}{|c|c|c|c|c|}
\hline 0 & 0 & 0 & 0 & 0 \\
\hline 0 & 0 & 0 & 0 & 0 \\
\hline-1 & -1 & 4 & -1 & -1 \\
\hline 0 & 0 & 0 & 0 & 0 \\
\hline 0 & 0 & 0 & 0 & 0 \\
\hline
\end{tabular}

\begin{tabular}{|c|c|c|c|c|}
\hline 0 & 0 & -1 & 0 & 0 \\
\hline 0 & 0 & -1 & 0 & 0 \\
\hline 0 & 0 & 4 & 0 & 0 \\
\hline 0 & 0 & -1 & 0 & 0 \\
\hline 0 & 0 & -1 & 0 & 0 \\
\hline
\end{tabular}

\begin{tabular}{|c|c|c|c|c|}
\hline-1 & 0 & 0 & 0 & 0 \\
\hline 0 & -1 & 0 & 0 & 0 \\
\hline 0 & 0 & 4 & 0 & 0 \\
\hline 0 & 0 & 0 & -1 & 0 \\
\hline 0 & 0 & 0 & 0 & -1 \\
\hline
\end{tabular}

\begin{tabular}{|c|c|c|c|c|}
\hline 0 & 0 & 0 & 0 & -1 \\
\hline 0 & 0 & 0 & -1 & 0 \\
\hline 0 & 0 & 4 & 0 & 0 \\
\hline 0 & -1 & 0 & 0 & 0 \\
\hline-1 & 0 & 0 & 0 & 0 \\
\hline
\end{tabular}

Figure 1. Laplacian Operators

\subsection{Weighted Median Filter:}

Weighted median filter differs from median filter in which the specified pixels within a local neighborhood are repeated a given number of times in the computation of the median value $[10,14]$. It ignores the largest and smallest values and it is same when the weights are equal to 1.

\subsubsection{Computation of Weighted Median:}

1. Sort points in window.

- Let $\mathrm{x}(1) \leq \mathrm{x}(2) \leq \cdots \leq \mathrm{x}(\mathrm{p})$ be the sorted values.

- Let a(1), a(2), ․ a a(p) be the corresponding weights. 
2. Find i* such that the following equations hold

$$
\begin{aligned}
a_{i *}+\sum_{i=1}^{i *-1} a_{(i)} & \geq \sum_{i=i *+1}^{p} a_{(i)} \\
\sum_{i=1}^{i *-1} a_{(i)} & \leq \sum_{i=i *+1}^{p} a_{(i)}+a_{i *}
\end{aligned}
$$

Then the value $\mathrm{x}\left(\mathrm{i}^{*}\right)$ is the weighted median value. The weights can be adjusted to yield the "best" filter.

\subsection{Adaptive Centre Weighted Median Filter:}

CWM filters are the simplest weighted median filters and the easiest to design and implement [12]. In a CWM filter, the center sample is assigned a larger weight, i.e., $\mathrm{w}(0,0)=$ $2 \mathrm{~K}+1$ where $\mathrm{K} \geq 0$, and all other non-zero weights are equal to one, i.e., $w(i, j)=1$ for $\mathrm{i}, \mathrm{j} \neq$ $0, \mathrm{~K}$ is a nonnegative integer. A CWM filter is completely specified by two parameters, the window size and the center weight. The filtering behavior of a CWM filter will thus be controlled by these two parameters and it preserves details effectively [15]. A sample CWM window is shown below.

$$
\bar{W}=\left(\begin{array}{ccccc}
1 & \ldots & 1 & \ldots & 1 \\
\vdots & \vdots & \vdots & \vdots & \vdots \\
1 & \ldots & 2 K+1 & \ldots & 1 \\
\vdots & \vdots & \vdots & \vdots & \vdots \\
1 & \ldots & 1 & \ldots & 1
\end{array}\right)
$$

\subsection{Tristate Median Filter:}

It is the combination of the standard median (SM) filter and the center weighted median (CWM) filter to determine whether a pixel is corrupted, before applying filtering unconditionally as shown in Figure 2. The output of the tristate median filter correspond to one of three possible states, namely the origin pixel value (i.e., the pixel is noise-free), the SM filtered output (i.e., the pixel is corrupted), or the CWM filtered output (i.e., the pixel is probably uncorrupted) as shown below [13].

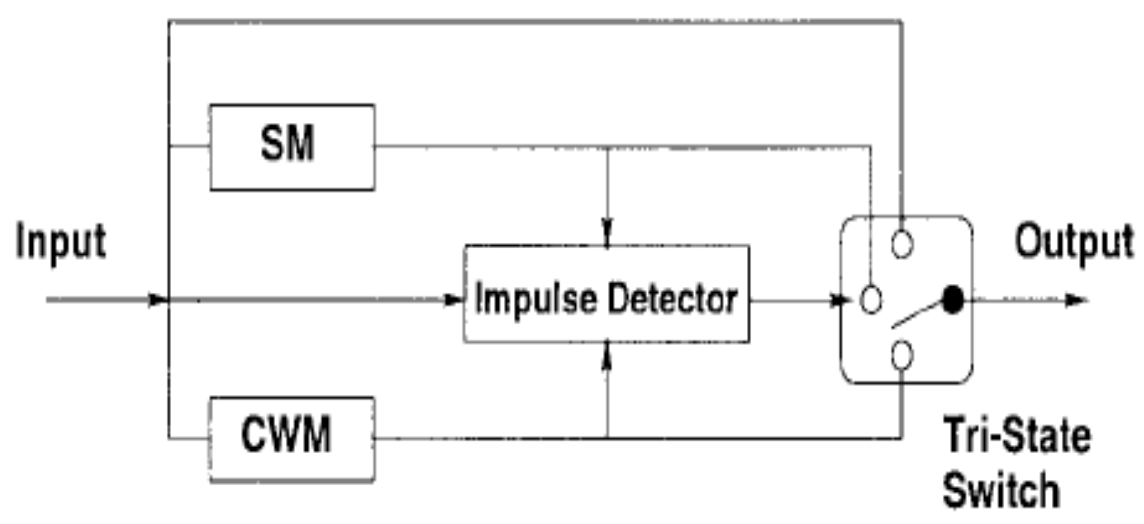

Figure 2. Tristate Median Filter 


$$
Y_{i j}^{\mathrm{TSM}}= \begin{cases}X_{i j} & T \geq d_{1} \\ Y_{i j}^{\mathrm{CWM}} & d_{2} \leq T<d_{1} \\ Y_{i j}^{\mathrm{SM}} & T<d_{2}\end{cases}
$$

where

$$
\begin{aligned}
& d_{1}=\left|X_{i j}-Y_{i j}^{\mathrm{SM} \overline{\mathrm{M}}}\right| \\
& d_{2}=\left|X_{i j}-Y_{i j}^{\mathrm{CWM}}\right| \quad d_{2} \leq d_{1}
\end{aligned}
$$

\section{Experimental Results and Discussion}

\subsection{Experimental Setup:}

The experiments have been carried out with MATLAB R2007b on a set of standard gray scale images. The impulse noise removal performance of the state of the art filters such as Median Filter [4], Adaptive Median Filter [6], switching median filter [7-9], weighted median filter [10], adaptive center-weighted median filter [11, 12], and tristate median filter[13] are compared in terms of MSE, PSNR and MSSIM.

\subsection{Image Quality Assessment Metrics:}

The image quality assessment measures are helpful in detecting the quality of the processed image in comparison with the original image. In our work, we concentrate on objective quality measurement like Mean Square Error (MSE), Peak Signal to Noise Ratio (PSNR) and Structural Similarity Measure (MSSIM) to evaluate the quality of the processed image.

\subsubsection{Mean Square Error:}

The most frequently used image quality measures are deviations between the original and processed images of which the mean square error (MSE) or signal to noise ratio (SNR) are the most common measures. The effectiveness of the algorithm stands in minimizing the mean square error [1]. If $\mathrm{F}(\mathrm{X}, \mathrm{Y})$ is the original clean image, $\mathrm{G}(\mathrm{X}, \mathrm{Y})$ is the corrupted image and $\mathrm{I}(\mathrm{X}, \mathrm{Y})$ is the denoised image then MSE is given by

$$
M S E=\frac{1}{M N} \sum_{X=1}^{M} \sum_{Y=1}^{N}(F(X, Y)-I(X, Y))^{2}
$$

\subsubsection{Peak Signal to Noise Ratio:}

Larger PSNR indicate a smaller difference between the original uncorrupted image and the denoised image. This is the most widely used objective image quality/distortion measure [1]. The main advantage of this measure is ease of computation. PSNR is calculated using,

$$
P S N R=20 \log _{10}\left(F_{\max } / \sqrt{M S E}\right)
$$

where $F_{\max }=255$ for an 8-bit image. 


\subsubsection{Multiscale Structural Similarity Index:}

Structural similarity provides an alternative and complementary approach to the problem of image quality assessment. It is based on the assumption that the human visual system (HVS) is highly adapted for extracting structural information from the scene, and therefore a measure of structural similarity should be a good approximation of perceived image quality. Multi-scale structural similarity method and introduced in [16, 17] is an image synthesisbased approach to calibrate the parameters that weight the relative importance between different scales. The original image is designated as scale 1 and the highest scale is $M$. The luminescence comparison $\mathrm{l}(\mathrm{x}, \mathrm{y})$ is calculated only at scale $\mathrm{M}$. At $\mathrm{j}^{\text {th }}$ scale, the contrast $\mathrm{c}(\mathrm{x}, \mathrm{y})$ and structural s(x,y) comparisons are calculated using

$$
\begin{array}{r}
l_{M}(x, y)=\frac{2 \mu_{x} \mu_{y}+C_{1}}{\mu_{x}^{2}+\mu_{y}^{2}+C_{1}} \\
c_{j}(x, y)=\frac{2 \sigma_{x} \sigma_{y}+C_{2}}{\sigma_{x}^{2}+\sigma_{y}^{2}+C_{2}} \\
s_{j}(x, y)=\frac{\sigma_{x y}+C_{3}}{\sigma_{x} \sigma_{y}+C_{3}}
\end{array}
$$

where $\mathrm{C} 1=\left(\mathrm{K}_{1} \mathrm{~L}\right)^{2}, \mathrm{C}_{2}=\left(\mathrm{K}_{2} \mathrm{~L}\right)^{2}$ and $\mathrm{C}_{3}=\mathrm{C}_{2} / 2$. $\mathrm{L}$ is the dynamic range of the image. $\mathrm{K}_{1}$ and $\mathrm{K}_{2}$ are two scalar constants equal to 0.01 and 0.03 respectively. The multiscale SSIM is calculated as

\subsection{Experimental Results and Discussion:}

$$
\operatorname{SSIM}(x, y)=\left[l_{M}(x, y)\right]^{\alpha_{M}} \cdot \prod_{j=1}^{M}\left[c_{j}(x, y)\right]^{\beta_{j}}\left[s_{j}(x, y)\right]^{\gamma_{j}}
$$

The experimental results are presented in Table 1. The comparison of MSE, PSNR and MSSIM are shown in Figure 3-Figure 5 at various noise densities for cameraman (256X256) image. Figure 6(a-c) depicts the comparison of MSE, PSNR and MSSIM at 30\% noise for Barbara (512X512) image. Also the sample denoised images are shown in Figure 7.

COMPARISON OF MEAN SQUARE ERROR AT VARIOUS NOISE DENSITIES

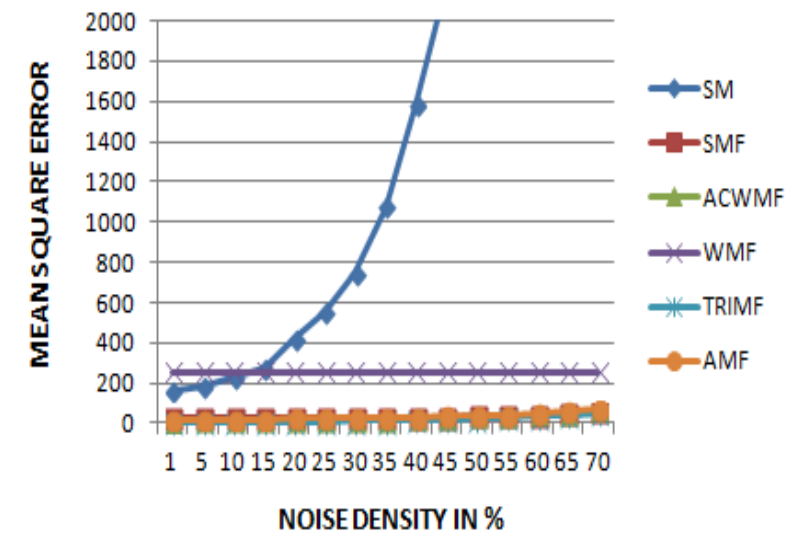

Figure 3. Comparison of MSE at Various Noise Densities 


\section{COMPARISON OF PEAK SIGNAL TO NOISE RATIO AT VARIOUS NOISE DENSITIES}

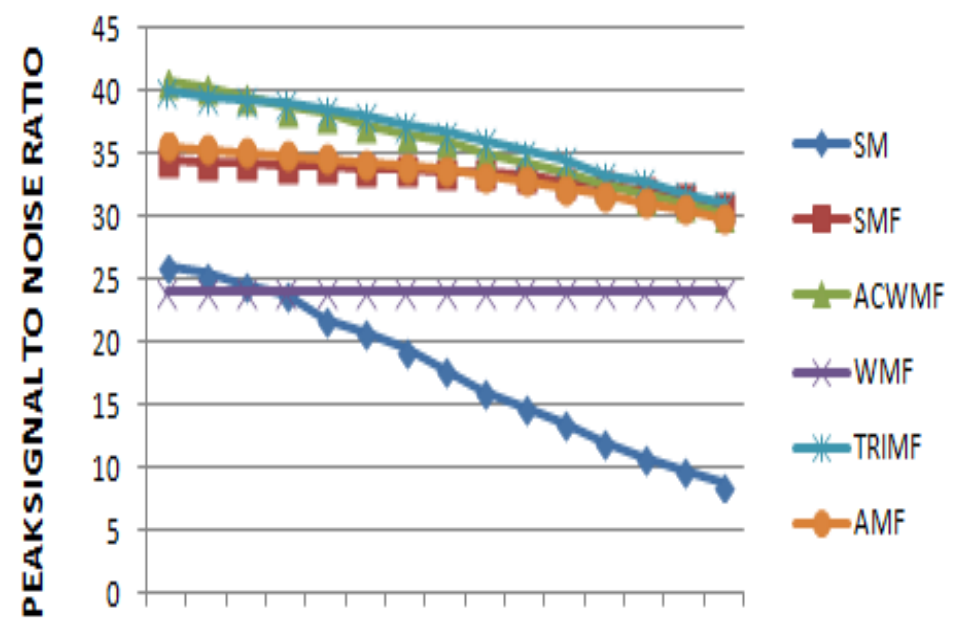

1510152025303540455055606570

NOISEDENSITY IN \%

Figure 4. Comparison of PSNR at Various Noise Densities COMPARISON OF MSSIM AT VARIOUS NOISE DENSITIES

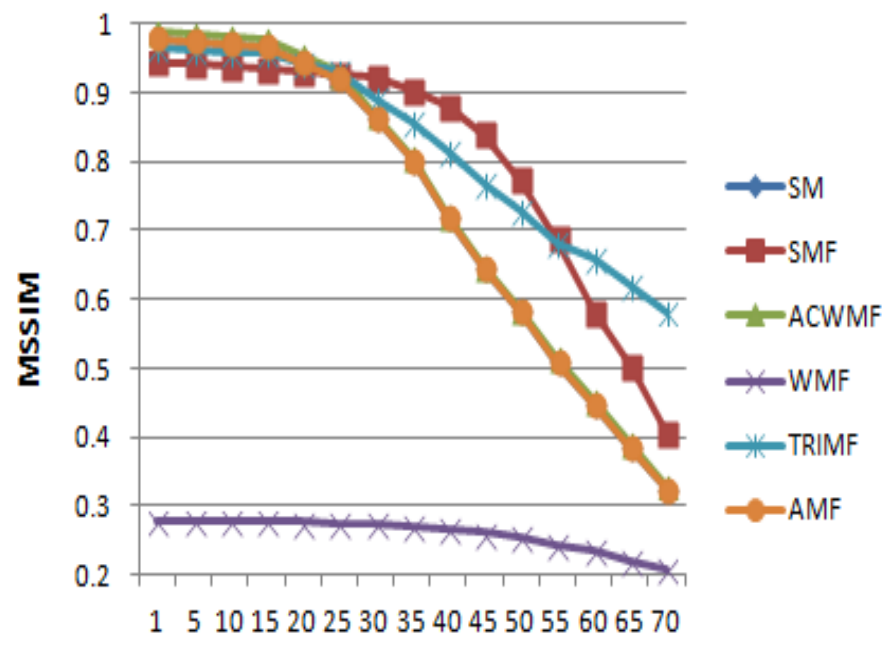

NOISEDENSITY IN \%

Figure 5. Comparison of MSSIM at Various Noise Densities 
International Journal of Signal Processing, Image Processing and Pattern Recognition Vol.6, No.5 (2013)

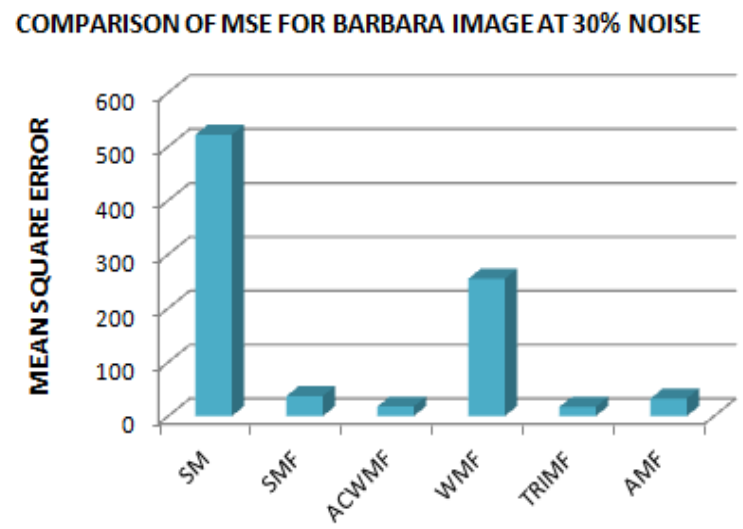

Figure 6 (a). Comparison of MSE at $30 \%$ Noise COMPARISON OF PSNR FOR BARBARA IMAGE AT 30\% NOISE

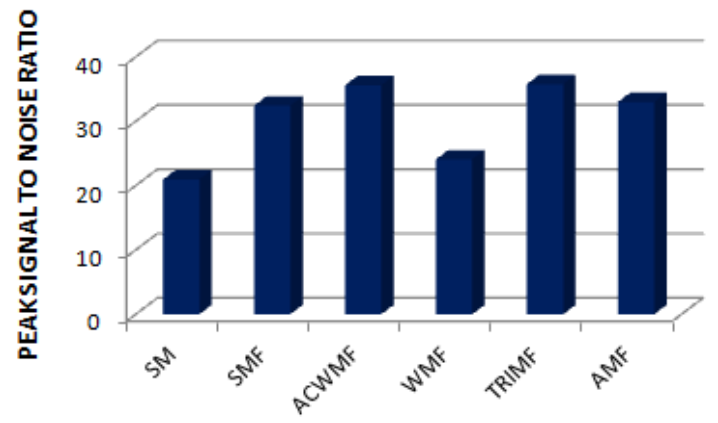

Figure 6 (b). Comparison of PSNR at $30 \%$ Noise COMPARISON OF MSSIM FOR BARBARA IMAGEAT 30\% NOISE

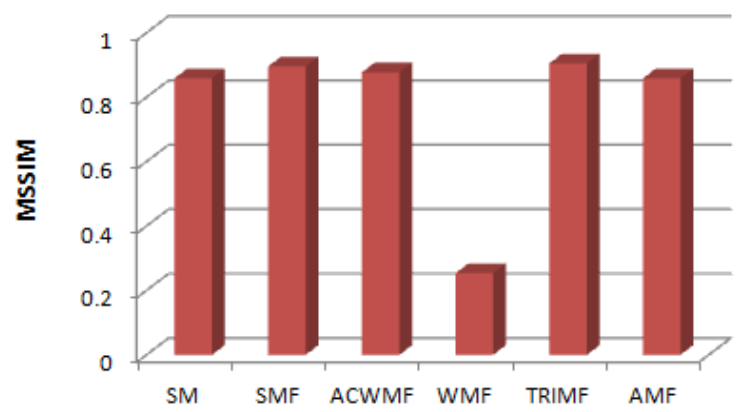

Figure 6 (c). Comparison of MSSIM at 30\% Noise 


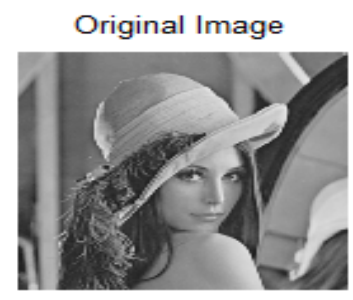

MF

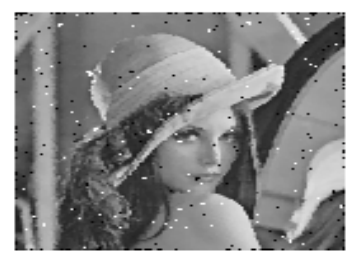

WMF

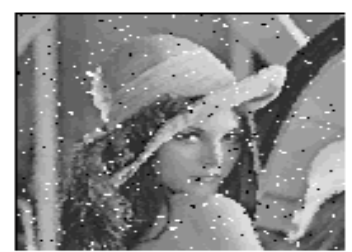

Noisy Image

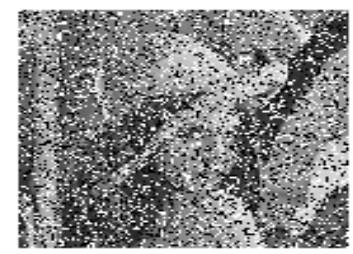

SMF

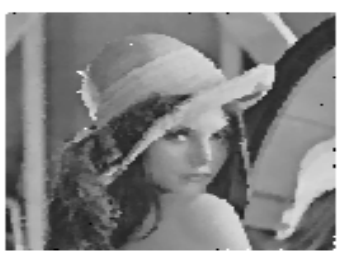

TMF

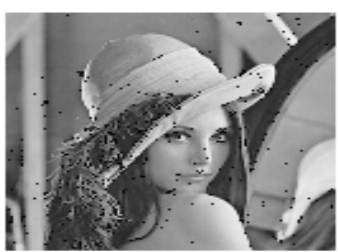

Added noise

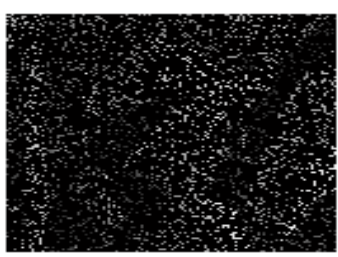

ACWMF

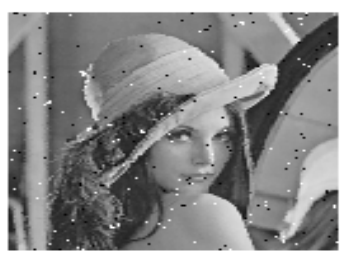

AMF

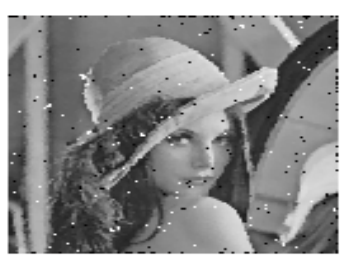

Figure 7. Comparison of MSSIM at $30 \%$ Noise

From the experimental results it is evident that, ACWMF and TRIMF exhibit similar performance which is superior to that of SM, SMF, WMF and AMF interms of MSE, PSNR and MSSIM. The denoised images resulting from tristate median filter is visually good among the methods compared. The SM, ACWMF and WMF lack in preserving edges and noise removal ability.

\section{Conclusion}

This paper presented an experimental analysis of median based impulse noise removal for gray scale images. Our experimental results show that, among the methods compared, tristate median filter and switching median filter exhibit visually appealing results. The other methods such as standard median filter, adaptive median filter, weighted median filter lack in preserving edges while retaining some noise components. However, these methods are suitable for impulse noise removal provided the noise density is low. If the noise density is too high, say $>90 \%$, then the methods like trimmed median [5] filter may yield better denoising performance.

\section{References}

[1] E. Jebamalar Leavline and S. Sutha, "Gaussian noise removal in gray scale images using fast Multiscale Directional Filter Banks”, Proceedings of International Conference on Recent Trends in Information Technology, Chennai, India, (2011) June 3-5.

[2] E. Jebamalar Leavline, S. Sutha and D. Asir Antony Gnana Singh, International Journal of Computer Applications, vol. 33, no. 10, (2011).

[3] S. Sutha, E. Jebamalar Leavline and D. Asir Antony Gnana Singh, European Journal of Scientific Research, vol. 86, no. 4, (2012).

[4] R. C. Gonzalez and R. E. Woods, "Digital Image Processing”, Pearson Prentice Hall Publication, (2008).

[5] E. Jebamalar Leavline and D. Asir Antony Gnana Singh, International Journal of Imaging \& Robotics, vol. 11, no. 3, (2013).

[6] H. Hwang and R. A. Haddad, IEEE Transactions on Image Processing, vol. 4, no. 4, (1995). 
[7] S. Zhang and M. A. Karim, IEEE Signal Processing Letters, vol. 9, no. 11, (2002).

[8] Z. Wang and D. Zhang, IEEE Transactions on Circuits and Systems II, vol. 46, no. 1, (1999).

[9] P.-E. Ng and M. A. Kai-Kuang, IEEE Transactions on Image Processing, vol. 15, no. 6, (2006).

[10] D. R. K. Brownrigg, Communications of the ACM, vol. 27, no. 8, (1984).

[11] J. Ko and Y. H. Lee, IEEE Trans. Circuits Syst., vol. 38, no. 9, (1991).

[12] T. Chen and H. R. Wu, IEEE Signal Process. Lett., vol. 8, no. 1, (2001).

[13] T. Chen, K.-K. Ma and L.-H. Chen, IEEE Transactions on Image Processing, vol. 8, no. 12, (1999).

[14] https://engineering.purdue.edu/ bouman/ece637/notes/pdf/NonLinFilter.pdf, Accessed online on (2013) 25 August.

[15] T. Sun, M. Gabbouj and Y. Neuvo, "Multidimensional Systems and Signal Processing”, vol. 6, no. 2, (1995).

[16] Z. Wang, E. P. Simoncelli and A. C. Bovik, "Multi-Scale Structural Similarity for Image Quality Assessment”, Proceedings of the $37^{\text {th }}$ IEEE Asilomar Conference on Signals, Systems and Computers, Pacific Grove, CA, (2003) November 9-12.

[17] Z. Wang, A. C. Bovik, H. R. Sheikh and E. P. Simoncelli, IEEE Transactions on Image Processing, vol. 13, no. 4, (2004).

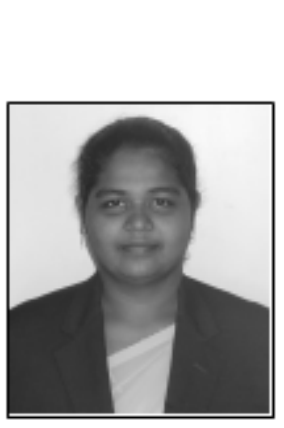

\section{Authors}

E.Jebamalar Leavline is with Department of Electronics and Communication Engineering, Bharathidasan Institute of Technology, Anna University, Tiruchirappalli, Tamil Nadu, India. She received her B.E, Electronics and Communication Engineering in 2005 and M.E in Applies Electronics in 2008 from Anna University, Chennai, Tamil Nadu, India. Also she is a M.B.A graduate in Education Management. She has published a good number of research papers in reputed International Journals and conferences. Her research interests includes image processing, signal processing, VLSI design, data mining, teaching learning process and engineering education. She is a Review committee member of several reputed journals and also she is a Member of ISTE and IAENG.

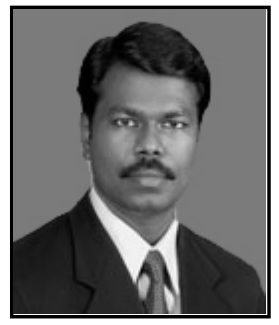

D.Asir Antony Gnana Singh is with Department of Computer Science and Engineering Bharathidasan Institute of Technology, Anna University, Tiruchirappalli, Tamil Nadu, India. He received his B.E and M.E degree in Computer Science and Engineering from Anna University, Chennai, Tamil Nadu, India in 2005 and 2008 respectively. He has published a good number of research papers in International Journals and conferences. His research interests are data mining, wireless networks, parallel computing, mobile computing, computer networks, image processing, software engineering, soft computing, teaching learning process and engineering education. He has participated and co-coordinated the faculty development programmes in India and abroad. He is a Review/Editorial committee member of several reputed journals and also he is a Member of ISTE, IET and IAENG. 